\title{
Megaesôfago secundário a miastenia grave em uma cadela da raça Pastor Alemão
}

\section{Megaesophagus secondary to myasthenia gravis in a female German shepherd dog}

\author{
Silvia Franco Andrade ${ }^{1 *}$; Rosa Maria Barilli Nogueira ${ }^{1}$; Alessandra Melchert ${ }^{1}$; \\ Maria Patrícia Chegança da Silva²; Yudney Pereira da Motta²; \\ Rejane Batista Brinholi ${ }^{3}$; Raimundo AlbertoTostes ${ }^{4}$; Osimar Sanches ${ }^{5}$
}

\section{Resumo}

Megaesôfago é uma causa comum de regurgitação sendo uma das possíveis etiologias a miastenia grave caracterizada por uma desordem neuromuscular que resulta em fraqueza dos músculos esqueléticos, do esôfago, da laringe e da faringe. O presente relato descreve um caso de uma cadela da raça Pastor Alemão, com histórico de fraqueza muscular e vômitos freqüentes, que foi diagnosticada como megaesôfago secundário à miastenia grave. A radiografia do animal apresentou um acentuado megaesôfago cervical e torácico. Após a instituição do protocolo terapêutico recomendado o animal apresentou melhora visível ao caminhar, porém as regurgitações pioraram e começou a apresentar tosse. Foi realizado novo exame radiográfico com presença, além de megaesôfago, de pneumonia aspirativa. A proprietária optou pela eutanásia do animal e a necropsia revelou severa pneumonia e dilatação acentuada da porção caudal do esôfago, caracterizando um megaesôfago de grande dimensão, colaborando com subsídios para um prognóstico desfavorável do caso.

Palavras-chave: Miastenia grave, megaesôfago, cão

\begin{abstract}
Megaesophagus is a common cause of regurgitation being one of the possible etiologies the myasthenia gravis characterized by a neuromuscular disorder that result in weakness of the skeletal muscles, of the esophagus, of the larynx and of the pharynx. The present report describes a case of a female German shepherd dog, with report of muscles weakness and frequent vomits, which was diagnosed as a megaesophagus secondary to myasthenia gravis. The radiograph of the animal presented an accentuated cervical and thoracic megaesophagus. After the institution of the recommended therapeutic protocol the animal presented visible improvement when walking, however the regurgitations worsened and it began to present cough. New radiograph was accomplished with presence, besides megaesophagus, of aspiration pneumonia. The owner opted for the euthanasia of the animal and the necropsy revealed severe pneumonia and an accentuated dilation of the posterior portion of the esophagus, collaborating with subsidies for an unfavorable prognostic of the case.
\end{abstract}

Key words: Myasthenia gravis, megaesophagus, dog

\footnotetext{
Professora Doutora do Departamento de Clínica Médica de Pequenos Animais do Curso de Medicina Veterinária da Universidade do Oeste Paulista (UNOESTE), Presidente Prudente, SP. E-mail:silviafranco@unoeste.br.

2 Residente da Clínica Médica de Pequenos Animais do Hospital Veterinário da UNOESTE.

3 Professora Mestre do Depto de Apoio Diagnóstico do Curso de Medicina Veterinária da UNOESTE.

4 Professor Doutor do Departamento de Anatomia Patológica da Faculdade de Medicina Veterinária do Centro Universitário de Maringá (CESUMAR), Maringá, PR

5 Professor Mestre do Depto de Anatomia Patológica do Curso de Medicina Veterinária da UNOESTE.

* Autor para correspondência
} 


\section{Introdução}

O megaesôfago pode ser definido como uma dilatação patológica do esôfago, porém se trata de um quadro mais complexo, que possui origens diversas, razão pela qual pode ser denominado de síndrome de megaesôfago (TORRES, 1997).

A afecção pode ser congênita ou adquirida e têm sido descritas predisposições hereditárias em algumas raças de cães, como nos Fox Terrier (MILLER et al., 1983), Schnauzer, Pastor Alemão, Dogue Alemão, Golden Retrevier e Setter Irlandês (GAYNOR; SHOFER; WASHABU, 1997). Em gatos é relativamente rara, talvez devido à prevalência de fibras musculares lisas no esôfago felino, sendo a raça Siamesa a mais predisposta (STURGESS et al., 2001). O diagnóstico é feito através dos sinais clínicos e achados radiográficos (WRAY; SPARKES, 2006).

A etiologia do megaesôfago congênito ainda é desconhecida, mas alguns autores sugerem que possa ser devido a uma falha sensorial ou uma lesão no centro de deglutição, mais especificamente na região medial da formação reticular lateral do tronco cerebral, ocorrendo, desta forma, problemas no peristaltismo esofágico em animais jovens (LEIB, 1983; TORRES, 1997).

O megaesôfago adquirido idiopático ocorre na idade adulta, sem antecedentes de problemas esofágicos, possuindo também etiologia desconhecida, porém, acomete principalmente animais que sofreram algum estresse importante, como fraturas, traumatismos (TORRES, 1997).

Já o megaesôfago adquirido secundário ocorre como conseqüência de causas primárias que provocam alterações motoras no esôfago ou no esfíncter gastro-esofágico, determinando uma dilatação passiva do mesmo (WRAY; SPARKES, 2006; GAYNOR; SHOFER; WASHABU, 1997). As principais causas de megaesôfago secundário são miastenia grave, lúpus eritematoso, polimiosite, polineurite, neuropatias degenerativas, hipoadrenocorticismo, hipotireoidismo, déficit de tiamina, intoxicações por metais pesados (chumbo e tálio), tumores (principalmente timoma) e problemas cervicais (TORRES, 1997).

A miastenia grave é uma das principais causas de megaesôfago adquirido secundário. Os principais sinais clínicos da miastenia grave são fraqueza muscular intensa, particularmente em membros anteriores, fadiga e ventroflexão da cabeça após o exercício, passos instáveis e curtos, podendo apresentar também regurgitação decorrente de megaesôfago e presença de bloqueio atrioventricular de $3^{0}$ grau (BAV $3^{0}$ grau) (HACKETT et al., 1995; YAM; SHELTON; SIMPSON, 1996).

A miastenia grave possui duas formas, a congênita não associada com a produção de anticorpos, e a forma adquirida que está relacionada com anticorpos produzidos contra os receptores de acetilcolina na junção neuromuscular (SHELTON; SCHULE; KASS, 1997). A forma congênita ocorre mais em Fox Terrier de pelo liso, Springer Spaniel, Samoiedas, Jack Russel e gatos siameses (MILLER et al., 1983; JOSEPH; CARRILLO; LENNON, 1988; YAM; SHELTON; SIMPSON, 1996). A provável etiologia desta patologia parece ser devido à redução no número de receptores colinérgicos presentes na membrana pós-sináptica (ODA et al., 1984). A forma adquirida prevalece mais nas raças Akita, Pastor Alemão, Fox Terrier, Jack Russel, Pointer, Chihuahuas, Rottweiler, Doberman Pinscher e Dálmata, e nos gatos Siameses, Abissínios e Somalis (SHELTON; SCHULE; KASS, 1997; NELSON; COUTO, 2006). Considera-se uma afecção de origem auto-imune onde são detectáveis anticorpos circulantes contra receptores de acetilcolina na junção neuromuscular (DEWEY et al, 1997).

O diagnóstico da miastenia grave se baseia nos sinais clínicos, na resposta positiva ao teste de administração de anticolinesterásico, na determinação de anticorpos circulantes ou por meio de métodos imunohistoquímicos, tanto em biopsia muscular quanto após a incubação do soro do paciente com músculo normal canino ou felino (DEWEY et al, 1997; SHELTON; SCHULE; KASS, 1997). 
A resposta positiva à administração de anticolinesterásico consiste na utilização de cloreto de edrofônio na dose de $0,1 \mathrm{a} 0,2 \mathrm{mg} / \mathrm{kg}$ IV ou neostigmina na dose de 1 a $2 \mathrm{mg}$ IM, que inibi a hidrólise enzimática de acetilcolina na junção neuromuscular, permitindo que a acetilcolina liberada dos terminais axônicos esteja disponível por um período mais prolongado e, portanto, com maior oportunidade de ligar-se a receptores musculares e iniciar um potencial de ação (DEWEY et al, 1997; ANDRADE, 2002; NELSON; COUTO, 2006). Deve-se tomar cuidado com a dosagem do teste, pois é possível desencadear uma crise de excesso colinérgico caracterizada por despolarização muscular excessiva, fraqueza, insuficiência respiratória, vômito e diarréia (NELSON; COUTO, 2006). A administração de atropina na dose de 0,05 a $0,2 \mathrm{mg} / \mathrm{kg}$ IM antes do teste é útil para prevenir a ocorrência destes efeitos indesejáveis (ANDRADE, 2002).

O tratamento consiste na administração de anticolinesterásicos de ação prolongada, como o brometo de piridostigmina na dose de $2 \mathrm{mg} / \mathrm{kg}$, a cada 8 ou 12 horas em cães, e na dose de $0,5 \mathrm{mg} / \mathrm{kg}$, a cada 12 horas em gatos. Tanto em cães como em gatos a dose deve ser individualizada com base na resposta clínica, sendo que animais com megaesôfago que não toleram a administração oral do anticolinesterásico pode ser necessário o tratamento com neostigmina na dose de 0,01 a $0,04 \mathrm{mg} / \mathrm{kg}$, intramuscular (IM), a cada 8 ou 6 horas. Em animais com miastenia grave adquirida pode ser necessário também o uso de imunossupressores como corticosteróides, além da terapia com anticolinesterásicos (YAM; SHELTON; SIMPSON, 1996; NELSON; COUTO, 2006).

\section{Relato do Caso}

Foi atendida uma cadela com 8 anos de idade, da raça Pastor Alemão, de nome Samanta, registrada sob o $\mathrm{n}^{0} 16900$, no setor de Clínica Médica de Pequenos Animais do Hospital Veterinário da UNOESTE, com histórico de fraqueza nos membros anteriores e vômitos freqüentes há três meses.

Ao exame físico contatou-se bom estado nutricional, parâmetros fisiológicos normais, porém o animal apresentava relutância em movimentar-se e ventroflexão do pescoço (Figura 1A). Foram realizados hemograma com pesquisa de hematozoário, dosagem de uréia e creatinina, com resultados dentro da normalidade. O exame radiográfico do tórax apresentou acentuado megaesôfago cervical e torácico com deslocamento ventral da traquéia e coração (Figura 1B). Com os sinais clínicos apresentados, fraqueza muscular generalizada associada a megaesôfago, raça (Pastor alemão), idade (8 anos), ausência de problemas dermatológicos e dor, suspeitou-se de miastenia grave com megaesôfago secundário.

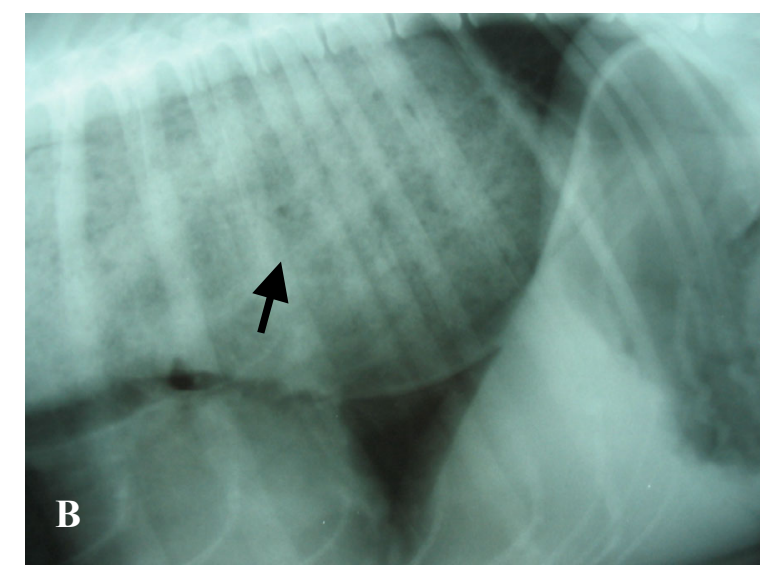

Figura 1. (A) Cadela apresentando fraqueza nos membros anteriores e ventroflexão da cabeça no momento da consulta; (B) Radiografia látero-lateral direita contrastada confirmando megaesôfago. 
Outras possíveis causas de megaesôfago como hipoadrenocorticismo, hipotireoidismo e lúpus eritematoso, foram descartadas por não apresentarem sinais compatíveis, além de serem causas incomuns de megaesôfago. Polimiosite, polimiopatia e polineuropatia também podem ser suspeitados e devem ser diagnósticos diferenciais de miastenia grave, através da realização de exame de eletromiografia e dosagem de anticorpos anti-receptores de acetilcolina (TAMS, 2005). Infelizmente, este último exame não é realizado no Brasil.

Foi realizado o protocolo de diagnostico terapêutico para miastenia grave com o uso de anticolinesterásico (neostigmina). Foi administrado sulfato de atropina $(0,2 \mathrm{mg} / \mathrm{kg}$ IM) 15 minutos antes da administração de neostigmina para prevenir efeitos muscarínicos. Após esses 15 minutos foi administrado neostigmina (Prostigmine $^{\circledR}$ ) na dose de 2 mg IM. Dez minutos após a administração do anticolinesterásico o animal já apresentava melhora evidente ao caminhar e conseguiu subir os degraus da escada sem relutância. Esse efeito durou por volta de 2 horas e 30 minutos, sendo, portanto confirmada a suspeita de miastenia grave.

Foi receitado brometo de piridostigmina $\left(\right.$ Mestinon $\left.^{\circledR}\right) 2 \mathrm{mg} / \mathrm{kg}$, a cada 12 horas, por via oral, prednisona $2 \mathrm{mg} / \mathrm{kg} / \mathrm{dia}$, por via oral, metoclopramida $0,5 \mathrm{mg} / \mathrm{kg}$, a cada 8 horas, por via oral e omeprazol $0,7 \mathrm{mg} / \mathrm{kg} / \mathrm{dia}$, por via oral, durante 10 dias. Orientouse que a alimentação do animal fosse preferencialmente pastosa e por gravidade, ou seja, em um plano mais elevado. Ao retorno, após 10 dias, a proprietária relatou que o animal apresentava melhora visível ao caminhar, porém as regurgitações haviam piorado e que a cadela apresentava tosse. Foi realizado novo exame radiográfico que constatou além do megaesôfago pré-existente, um padrão compatível com pneumonia aspirativa. A proprietária optou pela eutanásia do animal e o exame necroscópico revelou severa pneumonia e dilatação acentuada na porção caudal do esôfago, caracterizando o megaesôfago (Figura 2).
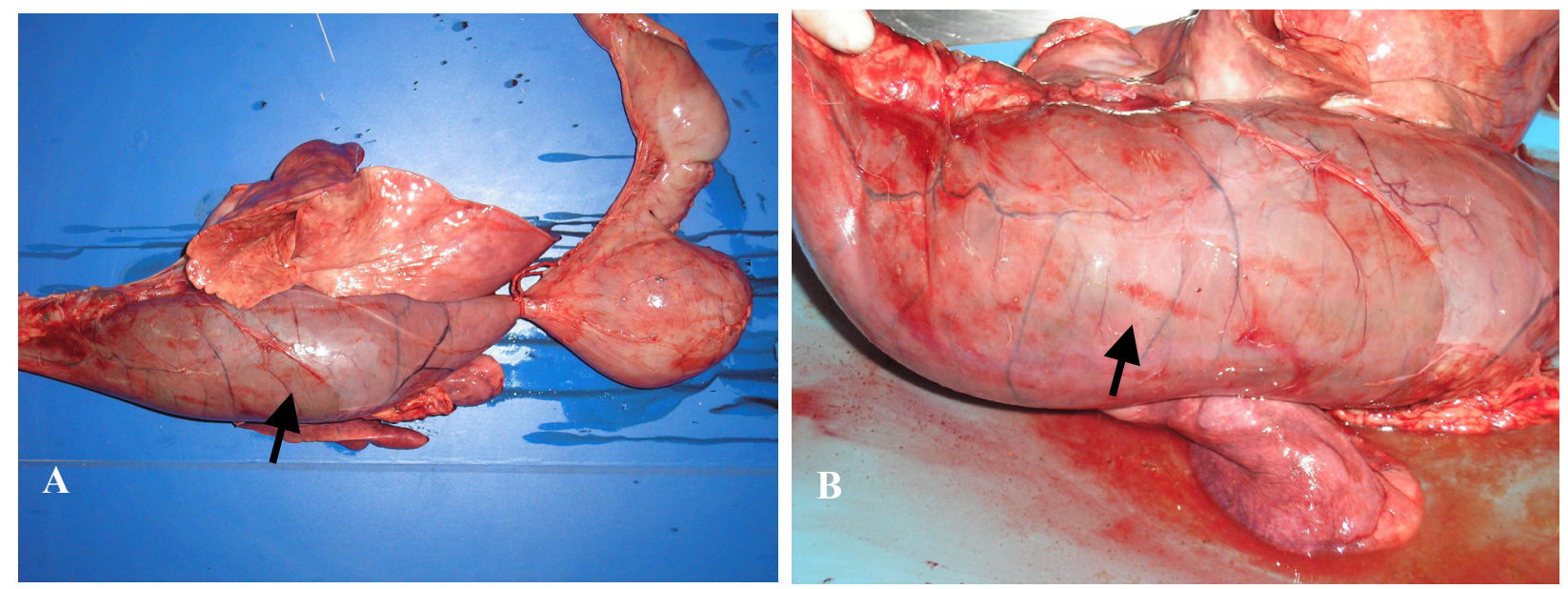

Figura 2. (A) Porção caudal do esôfago dilatado; (B) Detalhe do esôfago preenchido com água evidenciando a dilatação acentuada.

O presente relato está de acordo com outros descritos em cães com megaesôfago secundário à miastenia grave e com pneumonia aspirativa decorrente do processo esofágico (YAM; SHELTON; SIMPSON, 1996; KING; VITE, 1998).
O que chama atenção no presente caso é o tamanho exacerbado do esôfago oriundo da fraqueza muscular induzida pela miastenia grave, contribuindo com o prognóstico e a evolução clínica desfavorável para este animal. 


\section{Referências}

ANDRADE, S. F. Drogas que atuam no sistema nervoso periférico. In: - Manual de Terapêutica Veterinária. 2 ed. São Paulo: Roca, 2002. p.420-429.

DEWEY, C. W.; BAILEY, C. S.; SHELTON, G. D.; KASS, P. H.; CARDINET, G. H. Clinical forms of acquired myasthenia gravis in dogs: 25 cases (1988 - 1995). Journal of Veterinary Internal Medicine, Lakewood, v.11, n.2, p.50$57,1997$.

GAYNOR, A. R.; SHOFER, F. S.; WASHABAU, R. J. Risk factors for acquired megaesophagus in dogs. Journal of American Veterinary Medical Association, Schaumburg, v.211, n.11, p.1406-1412, 1997.

HACKETT, T. B.; VAN PELT, D. R.; WILLARD, M. D.; MARTIN, L. G.; SHELTON, G. D.; WINGFIELD, W. E. Third degree atrioventricular block and acquired myasthenia gravis in four dogs. Journal of American Veterinary Medical Association, Schaumburg, v.206, n.8, p.1173-1176, 1995.

JOSEPH, R. J.; CARRILLO, J. M.; LENNON, V. A. Myasthenia gravis in the cat. Journal of Veterinary Internal Medicine, Lakewood, v.2, n.2, p.75-79, 1988.

KING, L. G.; VITE, C. H. Acute fulminating myasthenia gravis in five dogs. Journal of American Veterinary Medical Association, Schaumburg, v.212, n.6, p.830-834, 1998.

LEIB, M. S. Megaesophagus in the dog, Part I, Anatomy, physiology and pathophysiology. Compendium of Continuous Education on Small Animal Practice, Auburn, v.5, n.10, p.825-833, 1983.

MILLER, L. M.; LENNON, V. A.; LAMBERT, E. H.; REED, S. M.; HEGREBERG, G. A.; MILLER, J. B.; OTT, R. L. Congenital myasthenia gravis in 13 smooth fox terriers. Journal of American Veterinary Medical Association, Schaumburg, v.182, n.7, p.694-697, 1983.
NELSON, R. W.; COUTO, C. C. Fundamentos de medicina interna de pequenos animais. 2.ed. Rio de Janeiro: Elsevier, 2006.

ODA, K.; LAMBERT, E.; LENNON, V. A.; PALMER, A. C. Congenital canine myasthenia gravis: deficient junctional acetylcholine receptors. Muscle and Nerve, Hoboken, v.7, n.9, p.705-716, 1984.

SHELTON, G. D.; SCHULE, A.; KASS, P. H. Risk factors for acquired myasthenia gravis in dogs:1,154 cases (1991 - 1995). Journal of American Veterinary Medical Association, Schaumburg, v.211, n.11, p.1428-1431, 1997.

STURGESS, C. P.; CANFIELD, P. J.; GRUFFYD-JONES, T. J.; STOKES, C. R. A gross and microscopical morphometric evaluation of feline large intestinal anatomy. Journal of Comparative Pathology, Exeter, v.124, n.4, p.255-264, 2001.

TAMS, T. R. Doenças do esôfago. In: TAMS, T. R. Gastroenterologia de pequenos animais. 2.ed. São Paulo: Roca, 2005.p.115-153.

TORRES, P. Megaesófago en el perro. Revisión bibliográfica y proposición de una nueva clasificación. Archivos de Medicina Veterinária, Valdivia, v.29, n.1, p.1323, 1997.

WRAY, J. D.; SPARKES, A. H. Use of radiographic measurements in distinguishing myasthenia gravis from other causes of canine megaoesophagus. Journal of Small Animal Practice, Oxford, v.47, n.5, p.256-263, 2006.

YAM, P. S.; SHELTON, G. D.; SIMPSON, J. W. Megaoesophagus secondary to acquired myasthenia gravis. Journal of Small Animal Practice, Oxford, v.37, n.4, p.179-183, 1996. 
